\title{
Kidney stone disease and cardiovascular events: a study on bidirectional causality based on mendelian randomization
}

\author{
Yuanyuan Zhao ${ }^{1,2,3,4 \#}$, Yang Fan ${ }^{5 \#}$, Mengru Wang ${ }^{6}$, Chenguang Yu ${ }^{6}$, Mengchen Zhou ${ }^{6}$, Dan Jiang ${ }^{6}$, \\ Dunfeng Du ${ }^{1,2,3,4}$, Shanshan Chen ${ }^{7}$ Xin Tu ${ }^{6}$
}

${ }^{1}$ Institute of Organ Transplantation, Tongji Hospital, Tongji Medical College, Huazhong University of Science and Technology, Wuhan, China; ${ }^{2}$ Key Laboratory of Organ Transplantation, Ministry of Education, Wuhan, China; ${ }^{3}$ NHC Key Laboratory of Organ Transplantation, Wuhan, China; ${ }^{4}$ Key Laboratory of Organ Transplantation, Chinese Academy of Medical Sciences, Wuhan, China; ${ }^{5}$ Department of Geriatrics, Tongji Hospital, Tongji Medical College, Huazhong University of Science and Technology, Wuhan, China; ${ }^{6}$ Key Laboratory of Molecular Biophysics of the Ministry of Education, College of Life Science and Technology, Center for Human Genome Research, Cardio-X Institute, Huazhong University of Science and Technology, Wuhan, China; ${ }^{7}$ Key Laboratory for Molecular Diagnosis of Hubei Province, The Central Hospital of Wuhan, Tongji Medical College, Huazhong University of Science and Technology, Wuhan, China

Contributions: (I) Conception and design: Y Zhao, D Du, X Tu; (II) Administrative support: X Tu, S Chen, D Du; (III) Provision of study materials or patients: Y Zhao, Y Fan; (IV) Collection and assembly of data: Y Zhao, Y Fan, M Wang, C Yu, D Jiang, M Zhou; (V) Data analysis and interpretation: Y Zhao, Y Fan; (VI) Manuscript writing: All authors; (VII) Final approval of manuscript: All authors.

\#These authors contributed equally to this work.

Correspondence to: Xin Tu. Key Laboratory of Molecular Biophysics of the Ministry of Education, College of Life Science and Technology, Center for Human Genome Research, Cardio-X Institute, Huazhong University of Science and Technology, Wuhan, China. Email: xtu@hust.edu.cn; Dunfeng Du. Institute of Organ Transplantation, Tongji Hospital, Tongji Medical College, Huazhong University of Science and Technology, Wuhan, China. Email: dfdu@tjh.tjmu.edu.cn; Shanshan Chen. Key Laboratory for Molecular Diagnosis of Hubei Province, The Central Hospital of Wuhan, Tongji Medical College, Huazhong University of Science and Technology, Wuhan, China. Email: chenshanshan@zxhospital.com.

Background: Kidney stone disease (KSD) has been reported to be associated with several cardiovascular diseases. However, the causality between the conditions remains unknown. In the study, we performed a study on bidirectional causality by two-sample Mendelian randomization (MR) to investigate the causality between KSD and cardiovascular diseases including coronary atherosclerosis, hypertension, and cardiomyopathy.

Methods: In the recent study, we performed a bidirectional two-sample MR study using available genomewide association summary data from the online database MRBASE. We identified genetic variants associated with KSD in one European population from UK Biobank (version 2, n=462,933). Two phenotypes of samples were chosen from the population to define our genetic instrumental variables: (I) samples with the phenotype of kidney stone/ureter stone/bladder stone (ukb-b-8297), and (II) samples with the phenotype of kidney stone surgery/lithotripsy (ukb-b-13537). For cardiovascular diseases, we picked up another independent European population from FinnGen Biobank $(n=93,421)$. We selected the exposure and outcome SNPs and then performed the two-sample MR using R package.

Results: After bidirectional causality by two-sample MR, we verified that genetic predisposition to KSD could increase the risk of coronary atherosclerosis (OR: $4.45 \times 1037$; SE $= \pm 7.80 \times 10^{14}, \mathrm{P}$ for MR-Egger $=0.024$ ) and cardiomyopathy (OR: $5.35 \times 10^{13}$; SE= $=7.18 \times 10^{6}$, P for IVW=0.045 for finn-a-I9_CARDMYO, and OR: $3.60 \times 10^{25} ; \mathrm{SE}= \pm 3.26 \times 10^{12}$, P for IVW=0.041 for finn-a-I9_CARDMYOOTH) when we used ukb-b-13537 as exposure group. Furthermore, hypertension could increase the risk of KSD (OR: 1.001; SE $= \pm 1.00$, P for IVW=0.003) when we used ukb-b-8297 as exposure group, without detected pleiotropy bias $(\mathrm{P}>0.05)$.

Conclusions: We confirmed KSD may trigger causal pathological processes including coronary atherosclerosis and cardiomyopathy. Furthermore, hypertension may causally affect KSD.

$\wedge$ ORCID: 0000-0003-0217-3290. 
Keywords: Kidney stone disease (KSD); coronary atherosclerosis; hypertension; cardiomyopathy; bidirectional Mendelian randomization

Submitted Oct 11, 2021. Accepted for publication Nov 26, 2021.

doi: $10.21037 /$ tau-21-899

View this article at: https://dx.doi.org/10.21037/tau-21-899

\section{Introduction}

Kidney stone disease (KSD) and cardiovascular diseases are two common conditions, and more and more studies reveal that KSD and cardiovascular diseases commonly coexist $(1,2)$. In addition, KSD is also associated with some metabolic disturbance such as obesity (3) and diabetes mellitus (4). However, there is little evidence about the causality between KSD and these systemic diseases. On the one hand, several studies suggested that occurrence of KSD could increase the risk of cardiovascular events such as hypertension $(5,6)$, coronary atherosclerosis $(7)$, and myocardial disorders (8). On the other hand, some study results showed that kidney stone formation was associated with subclinical coronary atherosclerosis (9) and so on. Therefore, the causality between KSD and cardiovascular events need to be determined, which has important implications for understanding the etiology of disease processes.

Mendelian randomization (MR) is a recently developed technique for causal inference based on large-scale genomewide association studies (GWAs) $(10,11)$. Briefly, MR is a technique that can detect causal effects of phenotypes as unbiased and possibly estimate their magnitude (11). This technique utilizes the random allocation of exposurerelated genetic variants. This random assortment minimizes the possibility of environmental confusion and enables the investigation to reveal the causal relationship between exposures and outcomes. Numerous studies have used MR to demonstrate the causality of coexisting phenotypes or diseases (12-14). Based on these, Hemani et al. developed a platform called MR-Base (http://app.mrbase.org/) that integrates a database of thousands of GWAS summary datasets with a web interface and $\mathrm{R}$ packages for automated causal inference through MR (15). Therefore, in the recent study, we performed two-sample MR by the database MRBASE to explore the bidirectional causality of KSD and cardiovascular events including three diseases: coronary atherosclerosis, hypertension, and cardiomyopathy.

\section{Methods}

\section{Ethical statement}

This research involving human participants were conformed to the guidelines set forth by the Declaration of Helsinki (2013).

\section{Implementation and operation}

In the recent study, we used two-sample MR designed (16) by the online database MRBASE (15) (http://app. mrbase.org/) which is a database and analytical platform for Mendelian randomization developed by the MRC Integrative Epidemiology Unit at the University of Bristol. All the data used were from genome-wide association studies (GWAS) and publicly available. These SNPs were selected based on the GWAS findings. We selected the exposure and outcome SNPs and then performed the twosample MR using R package.

\section{Study exposures and outcomes}

We selected the genetic variants of KSD in one European population from UK Biobank (version 2, n=462,933). Two phenotypes of samples were chosen to define our genetic instrumental variables: (I) samples with the phenotype of kidney stone/ureter stone/bladder stone (ukb-b-8297), and (II) samples with the phenotype of kidney stone surgery/lithotripsy (ukb-b-13537). For cardiovascular diseases, we detected three phenotypes including coronary atherosclerosis, hypertension, and cardiomyopathy. We chosen an independent European population from FinnGen Biobank to examine the causality between KSD and these cardiovascular diseases. The detailed sub-group ID were listed in Table 1. Both the databases were European population.

All the SNPs used us instrumental variants were from GWAS studies above. For SNPs selection, first of 
Table 1 Basic information of population in the study

\begin{tabular}{|c|c|c|c|}
\hline Phenotype & Group ID & Database & Description \\
\hline Kidney stone disease (KSD) & ukb-b-13537 & & $\begin{array}{l}\text { Operation code: percutaneous/open kidney stone surgery/ } \\
\text { lithotripsy }\end{array}$ \\
\hline Coronary atherosclerosis & finn-a-19_CORATHER & FinnGen Biobank & Coronary atherosclerosis \\
\hline \multirow[t]{2}{*}{ Cardiomyopathy } & finn-a-19_CARDMYO & FinnGen Biobank & Cardiomyopathy \\
\hline & finn-a-19_CARDMYOOTH & & Cardiomyopathy, other and unspecified \\
\hline
\end{tabular}

all, genetic variants associated with exposure factors at genome-wide significance $\left(\mathrm{P}<5 \times 10^{-5}\right)$ and not in linkage disequilibrium $\left(\mathrm{R}^{2}<0.01\right)$ were selected as instrumental SNPs. Furthermore, $\mathrm{SNP} \geq 0.05$ was defined as minor allele frequency. SNPs with MAF threshold was 0.01 . Second, through sensitivity analysis, the nonspecific SNPs were removed. Third, through checking the allele and frequency information of SNPs in both the exposure and outcome groups, we removed the SNPs with inconsistent information. We used "TwoSampleMR" R package to pick up appropriate variants.

\section{Statistical analysis (Mendelian randomization analysis)}

The primary analysis estimated the effect of exposure on the risk of outcome using "TwoSampleMR" R package. In the recent study, two complementary MR approaches were adopted in our analyses to assess the causal effect of the exposure on the outcome and its robustness, including MR Egger and Inverse variance weighted (IVW). Heterogeneity tests were performed using IVW and MR-Egger, and sensitivity analysis was performed using the weighted median method and Leave-one-out analysis which were described in the previous study (13). Scatter plots, forest plots, and funnel plots were used to present the results.

\section{Results}

\section{KSD increases the risk of coronary atherosclerosis}

We selected two subtype groups from UK Biobank with a genotype of KSD (ukb-b-8297 and ukb-b-13537) and one group with a genotype of coronary atherosclerosis from FinnGen Biobank (finn-a-I9_CORATHER) (Table 1). We first performed KSD $\rightarrow$ coronary atherosclerosis MR analysis. For exposure, 30 and 24 SNPs were included as genetic IVs for ukb-b-8297 and ukb-b-13537, respectively. For outcome, 28 SNPs were included. After analysis, the results showed that KSD increased the risk of coronary atherosclerosis using MR-Egger method (Figure 1A, OR: $\left.4.45 \times 10^{37} ; \mathrm{SE}= \pm 7.80 \times 10^{14}, \mathrm{P}=0.024\right)$ when ukb-b-8297 was treated as the exposure group, without detected pleiotropy bias $(\mathrm{P}>0.05)$. However, when we used ukb-b-13537 as exposure group, KSD didn't increased the risk of coronary atherosclerosis (Figure 1B, $\mathrm{P}>0.05$ ).

Then, we performed coronary atherosclerosis $\rightarrow \mathrm{KSD}$ MR analysis. For exposure, 19 SNPs were included as genetic IVs for finn-a-I9_CORATHER. For outcome, 12 and 10 SNPs were included ukb-b-8297 and ukb-b-13537, respectively. After analysis, the results showed that coronary atherosclerosis didn't affect the outcome of KSD (Figure $1 C, 1 D, \mathrm{P}>0.05)$. The $\mathrm{P}$ value in all the heterogeneity test results in the bidirectional MR analysis between KSD and coronary atherosclerosis was $>0.05$.

In conclusion, KSD increases the risk of coronary atherosclerosis, but coronary atherosclerosis didn't affect the outcome of KSD.

\section{KSD increases the risk of cardiomyopathy}

Two subtype groups from UK Biobank with the genotype of KSD (ukb-b-8297 and ukb-b-13537) and two groups with the genotype of cardiomyopathy from FinnGen Biobank (finn-a-I9_CARDMYO and finn-a-I9_CARDMYOOTH) were selected (Table 1). We first performed $\mathrm{KSD} \rightarrow$ cardiomyopathy MR analysis. For exposure, 30 and 24 SNPs were included as genetic IVs for ukb-b-8297 and ukb-b-13537, respectively. For outcomes, 28 and 28 SNPs were included for finn-a-I9_CARDMYO and finn-a-I9 
A

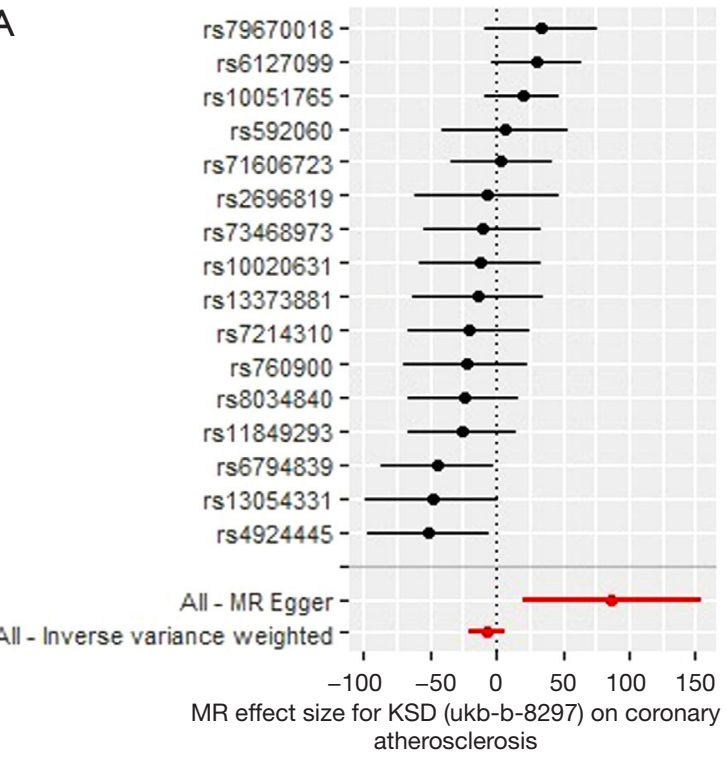

C

All - Inverse variance weighted -

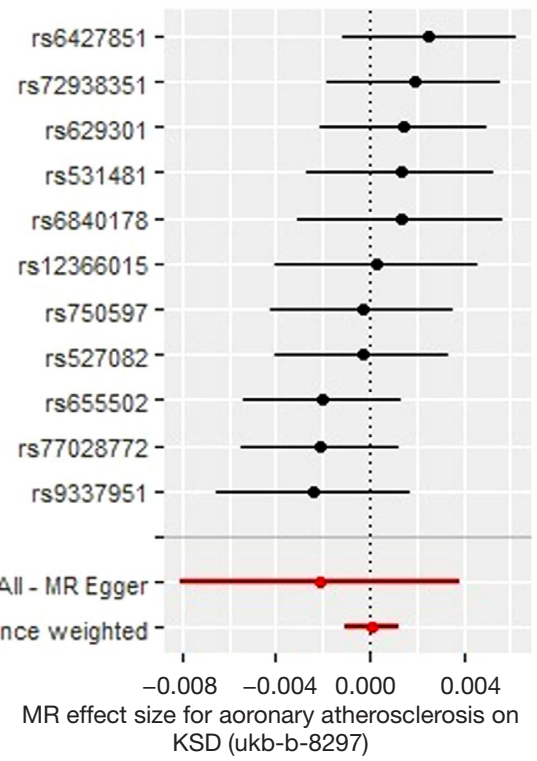

B

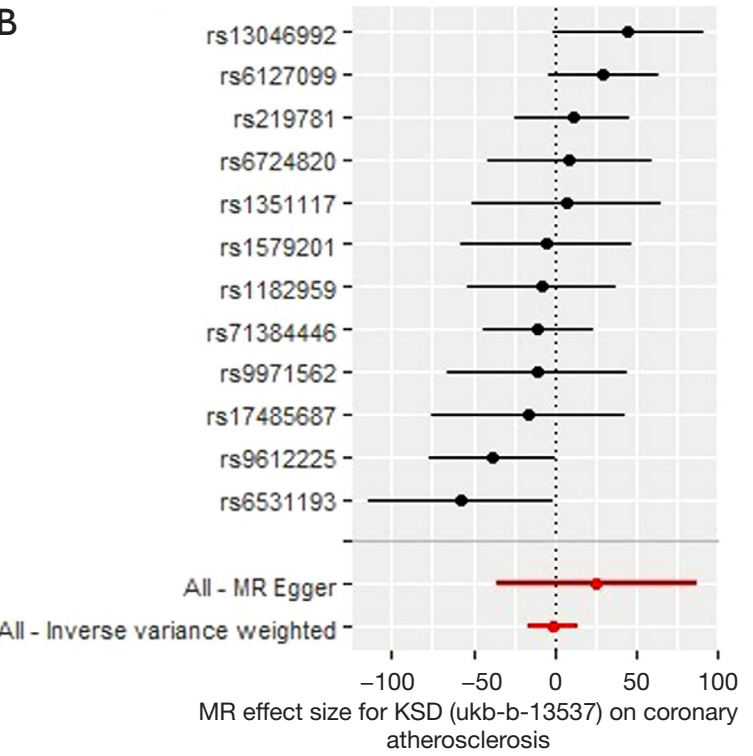

D

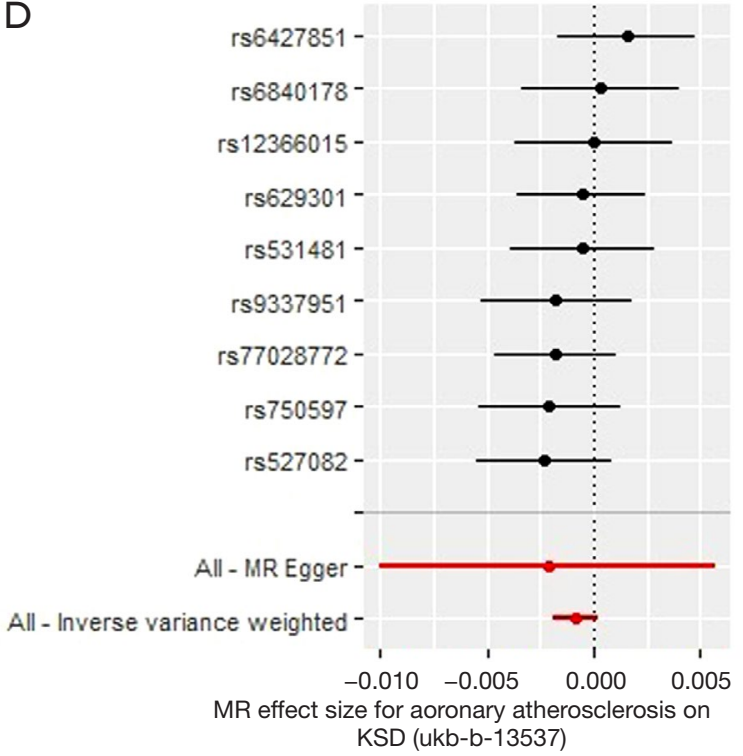

Figure 1 Mendelian randomization study of the bidirectional effect between KSD and coronary atherosclerosis. (A,B) Forest plots were presented for the MR effect size for KSD-ukb-b-8297 (A), and KSD-ukb-b-13537 (B) on coronary atherosclerosis. The results showed that KSD increased the risk of coronary atherosclerosis by using MR-Egger method (OR: 4.45x1037; $\mathrm{SE}= \pm 7.80 \times 1014, \mathrm{P}=0.024)$ when we used ukb-b-8297 but not ukb-b-13537 as exposure group. (C,D) Forest plots were presented for the MR effect size for coronary atherosclerosis on KSD-ukb-b-8297 (C) and KSD-ukb-b-13537 (D). The results showed that coronary atherosclerosis didn't affect the outcome of KSD ( $P>0.05)$. For the forest plots, red points showing the combined causal estimate using all SNPs together in a single instrument, using each of two different methods [inverse-variance weighted (IVW) and MR-Egger]. Horizontal lines denote $95 \%$ confidence intervals.

CARDMYOOTH, respectively. After analysis, the results showed that KSD increased the risk of cardiomyopathy by using IVW method [OR: $5.35 \times 10^{13} ; \mathrm{SE}= \pm 7.18 \times 10^{6}, \mathrm{P}=0.045$ for finn-a-I9_CARDMYO (Figure $2 A$ ), and OR: $3.60 \times 10^{25}$; $\mathrm{SE}= \pm 3.26 \times 10^{12}, \mathrm{P}=0.041$ for finn-a-I9_CARDMYOOTH
(Figure 2B)] when we used ukb-b-13537 as exposure group, without detected pleiotropy bias $(\mathrm{P}>0.05)$. However, when we used ukb-b-8297 as exposure group, KSD didn't increased the risk of cardiomyopathy (Figure $2 C$ for finn-a-I9_CARDMYO, and Figure 2D for finn-a-I9_ 
A

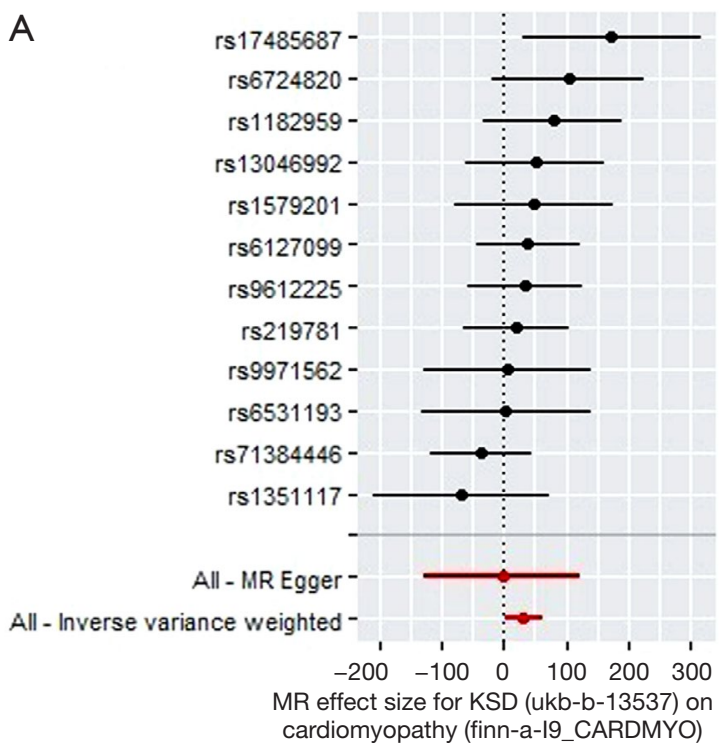

C

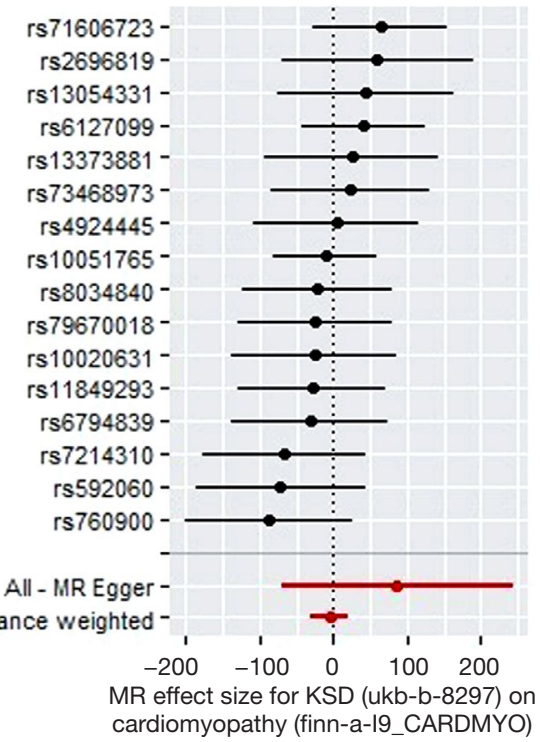

B

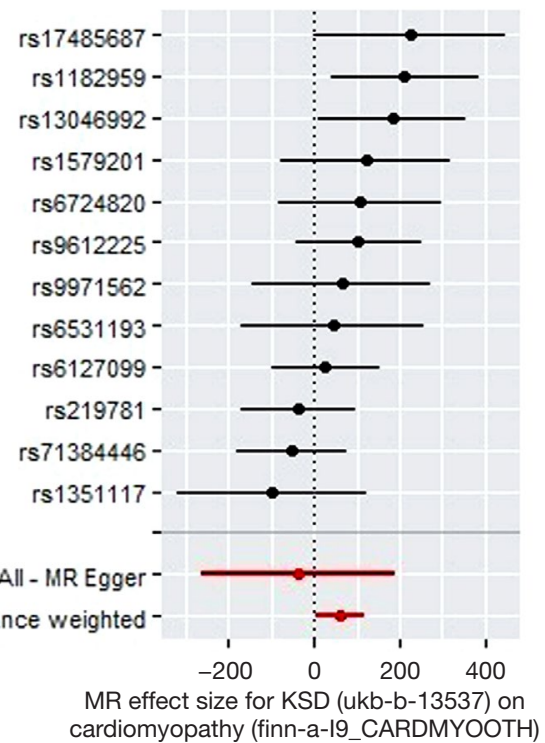

D

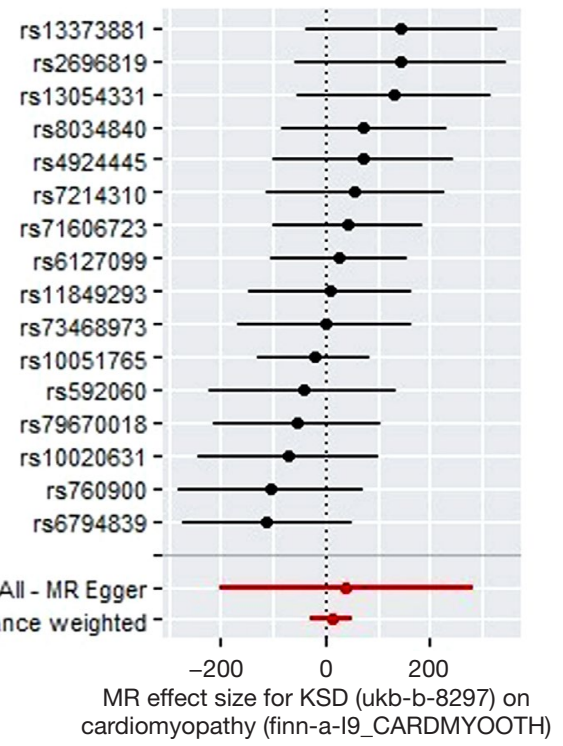

Figure 2 Mendelian randomization study of the effect of KSD on cardiomyopathy. (A,B) Forest plots were presented for the MR effect size for KSD-ukb-b-13537 on cardiomyopathy-finn-a-I9_CARDMYO (A) and cardiomyopathy-finn-a-I9_CARDMYOOTH (B). The results showed that KSD-ukb-13537 increased the risk of cardiomyopathy by using IVW method [OR: $5.35 \times 1013$; SE $= \pm 7.18 \times 106$, $\mathrm{P}=0.045$ for finn-a-I9_CARDMYO (A), and OR: 3.60x1025; SE= $\pm 3.26 \times 1012, \mathrm{P}=0.041$ for finn-a-I9_CARDMYOOTH (B)] when we used ukb-b-13537 as exposure group. (C,D) Forest plots were presented for the MR effect size for KSD-ukb-b-8297 on cardiomyopathy-finna-I9_CARDMYO (C) and cardiomyopathy-finn-a-I9_CARDMYOOTH (D). The results showed that KSD-ukb-b-8297 didn't affect the outcome of cardiomyopathy-finn-a-I9_CARDMYO (C) nor cardiomyopathy-finn-a-I9_CARDMYOOTH (D) (P>0.05). For the forest plots, red points showing the combined causal estimate using all SNPs together in a single instrument, using each of two different methods (inverse-variance weighted [IVW] and MR-Egger). Horizontal lines denote $95 \%$ confidence intervals.

\section{CARDMYOOTH, P>0.05).}

Then, we performed cardiomyopathy $\rightarrow$ KSD MR analysis. After analysis, the results showed that cardiomyopathy didn't affect the outcome of KSD (Figure S1, P>0.05). All the heterogeneity test results in the bidirectional MR analysis between KSD and coronary atherosclerosis were with $\mathrm{P}>0.05$.

In conclusion, KSD increases the risk of cardiomyopathy, 
but cardiomyopathy didn't affect the outcome of KSD.

\section{Hypertension increases the risk of KSD}

Two subtype groups from UK Biobank with genotype of KSD (ukb-b-8297 and ukb-b-13537) and three groups with genotype of hypertension from FinnGen Biobank (finn-a-I9_HYPTENS) (Table 1). We first performed KSD $\rightarrow$ hypertension MR analysis. For exposure, 30 and 24 SNPs were included as genetic IVs for ukb-b-8297 and ukb-b-13537, respectively. For outcome, 28 SNPs were included. After analysis, the results showed that KSD didn't affect the outcome of hypertension (Figure $3 A, 3 B, \mathrm{P}>0.05$ ). Then, we performed hypertension $\rightarrow$ KSD MR analysis. For exposure, 77 SNPs were included as genetic IVs for finn-a-I9_HYPTENS. For outcome, 47 and 41 SNPs were included ukb-b-8297 and ukb-b-13537, respectively. After analysis, the results showed that hypertension could increase the risk of KSD by using IVW method (Figure 3C, OR: 1.001; $\mathrm{SE}= \pm 1.00, \mathrm{P}=0.003$ ) when we used ukb-b-8297 as exposure group, without detected pleiotropy bias $(\mathrm{P}>0.05)$. However, when we used ukb-b-13537 as exposure group, hypertension didn't increase the risk of KSD (Figure 3D, P>0.05). All the heterogeneity test results in the bidirectional MR analysis between KSD and hypertension were with $\mathrm{P}>0.05$.

In conclusion, hypertension increases the risk of KSD, but KSD didn't affect the outcome of hypertension.

\section{Discussion}

In this MR-based bidirectional causality study, we detected the causality between KSD and cardiovascular events, including coronary atherosclerosis, hypertension, and cardiomyopathy. We found that KSD was associated with higher coronary atherosclerosis and cardiomyopathy risks, and hypertension was associated with a higher KSD risk. In the bidirectional MR analysis between KSD and cardiovascular events, all the heterogeneity test results were with $\mathrm{P}>0.05$, and no detected pleiotropy bias was detected $(\mathrm{P}>0.05)$. Based on a wide variety of sensitivity analyses to test the MR assumptions, our results showed an association between KSD and cardiovascular events are likely to be causal. Moreover, our findings are consistent with previous observational studies $(7-9,17,18)$. Lots of observational studies proved that KSD and cardiovascular diseases commonly coexisted. But the causality between these phenotypes couldn't be known from these studies. The main benefit of MR studies is to avoid the common biases in the conventional observational literature (19). Based on these, we thought our findings were useful for patient care. We recommend that if patients with KSD, regular image logical examinations were suggested to monitor the probable risk of coronary atherosclerosis and cardiomyopathy. Meanwhile, for patients with hypertension, KSD was also need to be noticed in patient care.

In the year of 1976, Elmfeldt et al. first reported an association between KSD and cardiovascular events (20). However, this cross-sectional study had only included 299 males and adjusted for age. Subsequently, more and more studies observed the association. In our study, we focused on three common cardiovascular events: coronary atherosclerosis, hypertension, and coronary atherosclerosis. Our study first confirmed the causality between these three clinical phenotypes and KSD. For hypertension and KSD, although several studies presented an increased risk of hypertension with KSD (21-23), our MR study showed that patients with KSD didn't get a higher risk of hypertension $(\mathrm{P}>0.05)$. This maybe because certain types of stones are associated with different comorbidities. Nevertheless, hypertension might be one independent cause of kidney stone formation (OR: 1.001; $\mathrm{SE}= \pm 1.00$, $\mathrm{P}=0.003)$. Cupisti and colleagues believed that the relationship between hypertension and KSD had similar pathophysiological mechanisms to insulin resistance in diabetes (24). KSD is generally recognized as a systemic disease indicating an adverse metabolic environment (4). Therefore, hypertension which was a systematic disease could contribute to kidney stone formation. In addition, hypercalciuria, which is usually described in hypertension, can increase the urinary calcium excretion and may cause the formation of calcium kidney stones (24-26). However, it was worth mentioning that in our recent study, we only observed that hypertension could increase the risk of KSD when we used ukb-b-8297 as an exposure group based on an OR of 1.001. When we used ukb-b-13537 as an exposure group, hypertension didn't increase the risk of KSD $(\mathrm{P}>0.05)$. Based on these results, we thought that the observed statistical and clinical significance was not remarkable. More independent analysis need to be performed to validate the findings in future studies. In addition, through the bidirectional MR study, we confirmed that KSD was associated with a higher coronary atherosclerosis ( $\mathrm{P}$ for MR-Egger $=0.024$ ) and cardiomyopathy ( $\mathrm{P}$ for IVW=0.045 for finna-I9_CARDMYO group, and P for IVW=0.041 for 

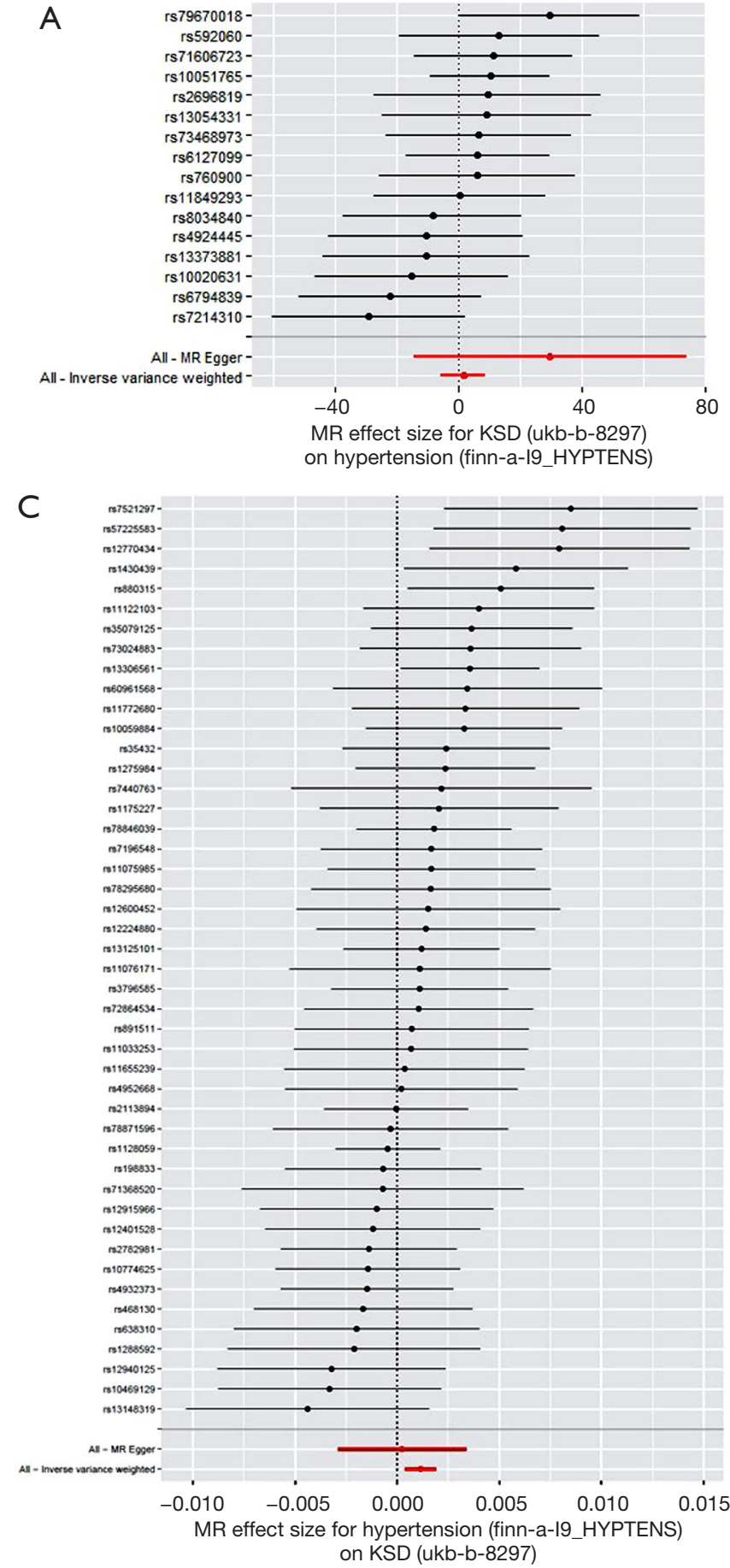

B

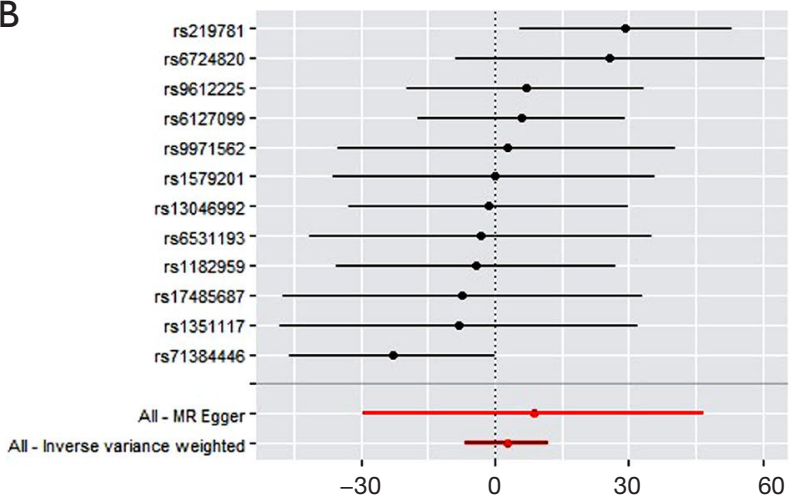

MR effect size for KSD (ukb-b-13537) on hypertension (finn-a-19_HYPTENS)

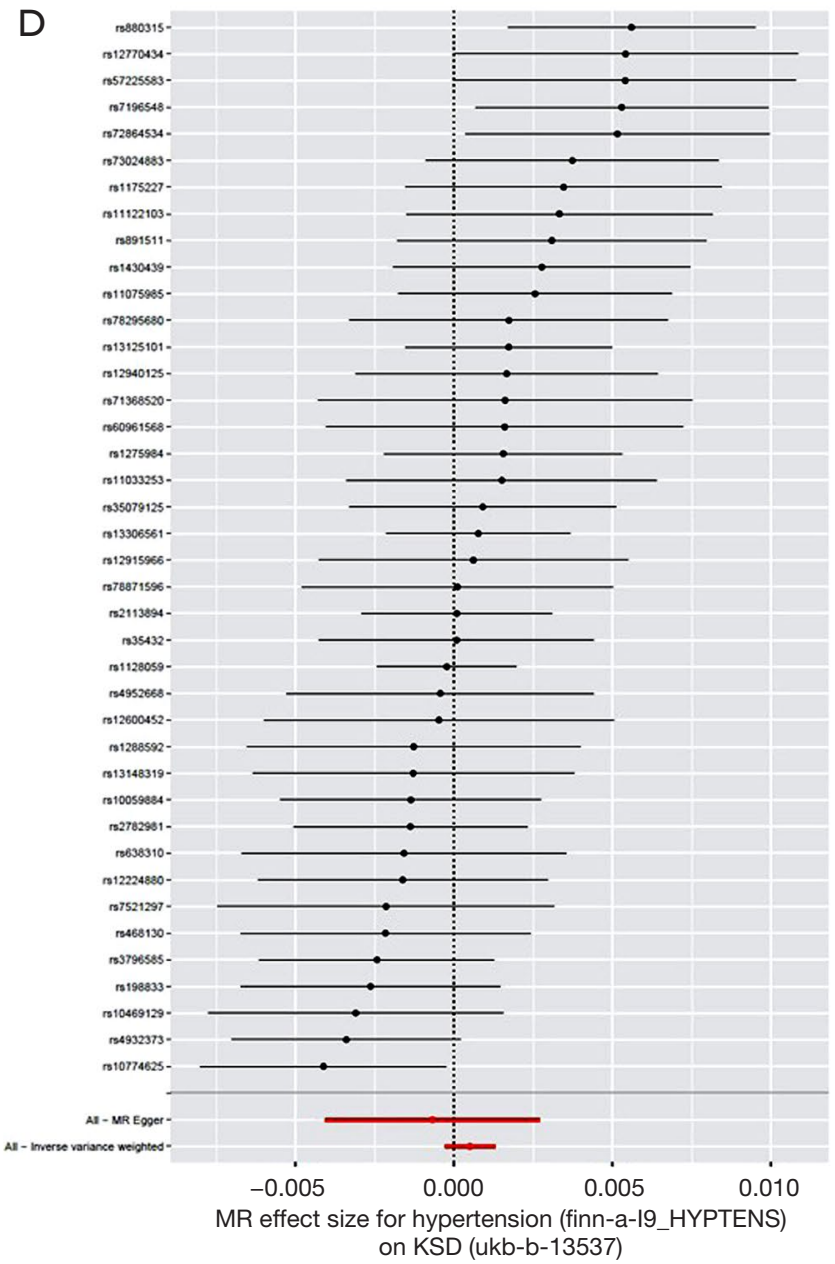

Figure 3 Mendelian randomization study of the bidirectional effect between KSD and hypertension. (A,B) Forest plots were presented for the MR effect size for KSD-ukb-b-8297 (A), and KSD-ukb-b-13537 (B) on hypertension. The results showed that KSD didn't affect the outcome of hypertension $(\mathrm{P}>0.05)$. (C,D) Forest plots were presented for the MR effect size for hypertension on KSD-ukb-b-8297 (C) and KSD-ukb-b-13537 (D). The results showed that hypertension could increase the risk of KSD by using IVW method (C, OR: 1.001; SE= $\pm 1.00, \mathrm{P}=0.003)$ when we used ukb-b-8297 as exposure group. However, when we used ukb-b-13537 as exposure group, hypertension didn't increased the risk of KSD (D, $\mathrm{P}>0.05$ ). For the forest plots, red points showing the combined causal estimate using all SNPs together in a single instrument, using each of two different methods [inverse-variance weighted (IVW) and MR-Egger]. Horizontal lines denote 95\% confidence intervals. 
finn-a-I9_CARDMYOOTH group) risk. Of course, we need to pay attention to the that we only observed the significance between KSD and cardiomyopathy when we used ukb-b-13537 as an exposure group. We thought the source of samples in the two groups (ukb-b-13537 and ukb-b-8297) were different which cause to the different results. Some studies thought that patients with kidney stones have an increased pulse-wave velocity, arterial stiffness and vascular calcifications $(27,28)$. These factors were associated with cardiovascular events such as coronary atherosclerosis. Furthermore, most kidney stones are composed of calcium oxalate, which can accumulate in all tissues, including the heart, and even lead to heart tissues damage in some reported cases $(29,30)$. In these above cases, patients with Primary hyperoxaluria, a rare disease with a genetic disorder characterized by increased urinary excretion of calcium oxalate, accompanied by cardiomyopathy. Our study presented that other types of kidney stones could also increase the risk of cardiomyopathy.

There is a limitation with the present study. First, we didn't find appropriate data to verify our results in other populations such as Asians. Second, the effect on hypertension for KSD, and on KSD for coronary atherosclerosis were validated in only one group. Third, for $\mathrm{KSD}$, we checked the population information carefully, and unfortunately, we found that these KSD patients were only with a diagnosis of kidney stone/ureter stone/bladder stone without the specific type of stone. Therefore, we could not confirm the specific type of KSD which could increase the risk of coronary atherosclerosis and cardiomyopathy. Based on these, we will collect another independent population to validate the findings in the future studies.

In conclusions, we confirmed KSD may trigger causal pathological processes including coronary atherosclerosis and cardiomyopathy. Furthermore, hypertension may causally affect KSD. Through a bidirectional MR study based on GWAS data, we attempted to discuss and reveal the causality between KSD and common cardiovascular events. Furthermore, these results proved that KSD play a crucial role to affect the pathology process of cardiovascular events.

\section{Acknowledgments}

Funding: This work was supported by grants from the National Natural Science Foundation of China (Nos. 81700300 and 81800296) and Wuhan Municipal Health
Commission Foundation (wx21Q38).

\section{Footnote}

Peer Review File: Available at https://dx.doi.org/10.21037/ tau-21-899

Conflicts of Interest: All authors have completed the ICMJE uniform disclosure form (available at https://dx.doi. org/10.21037/tau-21-899). The authors have no conflicts of interest to declare.

Ethical Statement: The authors are accountable for all aspects of the work in ensuring that questions related to the accuracy or integrity of any part of the work are appropriately investigated and resolved. This research involving human participants were conformed to the guidelines set forth by the Declaration of Helsinki (2013).

Open Access Statement: This is an Open Access article distributed in accordance with the Creative Commons Attribution-NonCommercial-NoDerivs 4.0 International License (CC BY-NC-ND 4.0), which permits the noncommercial replication and distribution of the article with the strict proviso that no changes or edits are made and the original work is properly cited (including links to both the formal publication through the relevant DOI and the license). See: https://creativecommons.org/licenses/by-nc-nd/4.0/.

\section{References}

1. Ferraro PM, Taylor EN, Eisner BH, et al. History of kidney stones and the risk of coronary heart disease. JAMA 2013;310:408-15.

2. Alexander RT, Hemmelgarn BR, Wiebe N, et al. Kidney stones and cardiovascular events: a cohort study. Clin J Am Soc Nephrol 2014;9:506-12.

3. Taylor EN, Stampfer MJ, Curhan GC. Obesity, weight gain, and the risk of kidney stones. JAMA 2005;293:455-62.

4. Taylor EN, Stampfer MJ, Curhan GC. Diabetes mellitus and the risk of nephrolithiasis. Kidney Int 2005;68:1230-5.

5. Madore F, Stampfer MJ, Rimm EB, et al. Nephrolithiasis and risk of hypertension. Am J Hypertens 1998;11:46-53.

6. Madore F, Stampfer MJ, Willett WC, et al. Nephrolithiasis and risk of hypertension in women. Am J Kidney Dis 1998;32:802-7.

7. Reiner AP, Kahn A, Eisner BH, et al. Kidney stones and subclinical atherosclerosis in young adults: the CARDIA 
study. J Urol 2011;185:920-5.

8. Rule AD, Roger VL, Melton LJ 3rd, et al. Kidney stones associate with increased risk for myocardial infarction. J Am Soc Nephrol 2010;21:1641-4.

9. Hsi RS, Spieker AJ, Stoller ML, et al. Coronary Artery Calcium Score and Association with Recurrent Nephrolithiasis: The Multi-Ethnic Study of Atherosclerosis. J Urol 2016;195:971-6.

10. Smith GD, Ebrahim S. 'Mendelian randomization': can genetic epidemiology contribute to understanding environmental determinants of disease. Int J Epidemiol 2003;32:1-22.

11. Davey Smith G, Hemani G. Mendelian randomization: genetic anchors for causal inference in epidemiological studies. Hum Mol Genet 2014;23:R89-98.

12. Hyman MC, Levin MG, Gill D, et al. Genetically Predicted Blood Pressure and Risk of Atrial Fibrillation. Hypertension 2021;77:376-82.

13. Sun D, Zhou T, Heianza Y, et al. Type 2 Diabetes and Hypertension. Circ Res 2019;124:930-7.

14. Malik R, Georgakis MK, Vujkovic M, et al. Relationship Between Blood Pressure and Incident Cardiovascular Disease: Linear and Nonlinear Mendelian Randomization Analyses. Hypertension 2021;77:2004-13.

15. Hemani G, Zheng J, Elsworth B, et al. The MR-Base platform supports systematic causal inference across the human phenome. Elife 2018;7:34408.

16. Burgess S TSG. Mendelian Randomization: Methods for Using Genetic Variants in Causal Estimation. London: Chapman and Hall/CRC, 2015.

17. Domingos F, Serra A. Nephrolithiasis is associated with an increased prevalence of cardiovascular disease. Nephrol Dial Transplant 2011;26:864-8.

18. Hamano S, Nakatsu H, Suzuki N, et al. Kidney stone disease and risk factors for coronary heart disease. Int J Urol 2005;12:859-63.

19. Dimitrakopoulou VI, Tsilidis KK, Haycock PC, et al. Circulating vitamin D concentration and risk of

Cite this article as: Zhao Y, Fan Y, Wang M, Yu C, Zhou M, Jiang D, Du D, Chen S, Tu X. Kidney stone disease and cardiovascular events: a study on bidirectional causality based on mendelian randomization. Transl Androl Urol 2021;10(12):4344-4352. doi: 10.21037/tau-21-899 seven cancers: Mendelian randomisation study. BMJ 2017;359:j4761.

20. Elmfeldt D, Vedin A, Wilhelmsson C, et al. Morbidity in representative male survivors of myocardial infarction compared to representative population samples. J Chronic Dis 1976;29:221-31.

21. Borghi L, Meschi T, Guerra A, et al. Essential arterial hypertension and stone disease. Kidney Int 1999;5 5:2397-406.

22. Gillen DL, Coe FL, Worcester EM. Nephrolithiasis and increased blood pressure among females with high body mass index. Am J Kidney Dis 2005;46:263-9.

23. Strazzullo P, Barba G, Vuotto P, et al. Past history of nephrolithiasis and incidence of hypertension in men: a reappraisal based on the results of the Olivetti Prospective Heart Study. Nephrol Dial Transplant 2001;16:2232-5.

24. Cupisti A, D'Alessandro C, Samoni S, et al. Nephrolithiasis and hypertension: possible links and clinical implications. J Nephrol 2014;27:477-82.

25. Coe FL, Evan AP, Worcester EM, et al. Three pathways for human kidney stone formation. Urol Res 2010;38:147-60.

26. Worcester EM, Coe FL. Clinical practice. Calcium kidney stones. N Engl J Med 2010;363:954-63.

27. Fabris A, Ferraro PM, Comellato G, et al. The relationship between calcium kidney stones, arterial stiffness and bone density: unraveling the stone-bone-vessel liaison. J Nephrol 2015;28:549-55.

28. Shavit L, Girfoglio D, Vijay V, et al. Vascular calcification and bone mineral density in recurrent kidney stone formers. Clin J Am Soc Nephrol 2015;10:278-85.

29. Bogle MA, Teller CF, Tschen JA, et al. Primary hyperoxaluria in a 27-year-old woman. J Am Acad Dermatol 2003;49:725-8.

30. Arat N, Akyıldız M, Tellioğlu G, et al. Cardiac involvement of primary hyperoxaluria accompanied by non-compaction cardiomyopathy and patent ductus arteriosus. Turk Kardiyol Dern Ars 2015;43:288-91. 


\section{Supplementary}

A

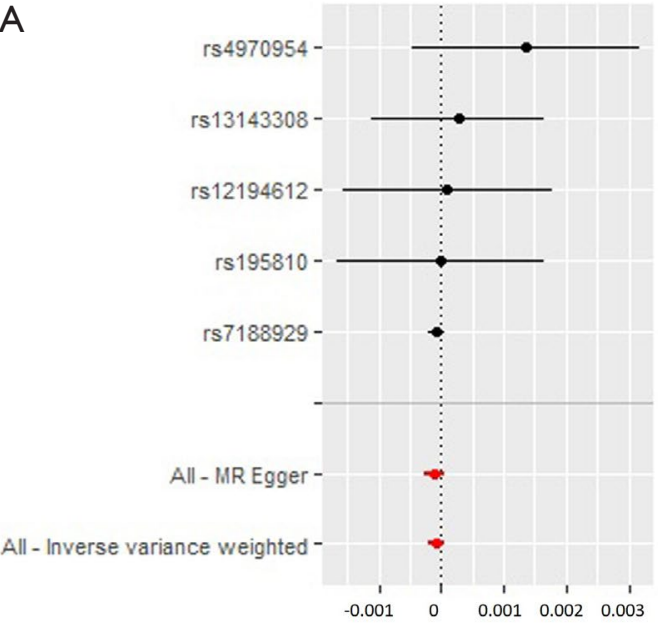

MR effect size for cardiomyopathy (finn-a-I9_CARDMYO) on KSD (ukb-b-8297)

C

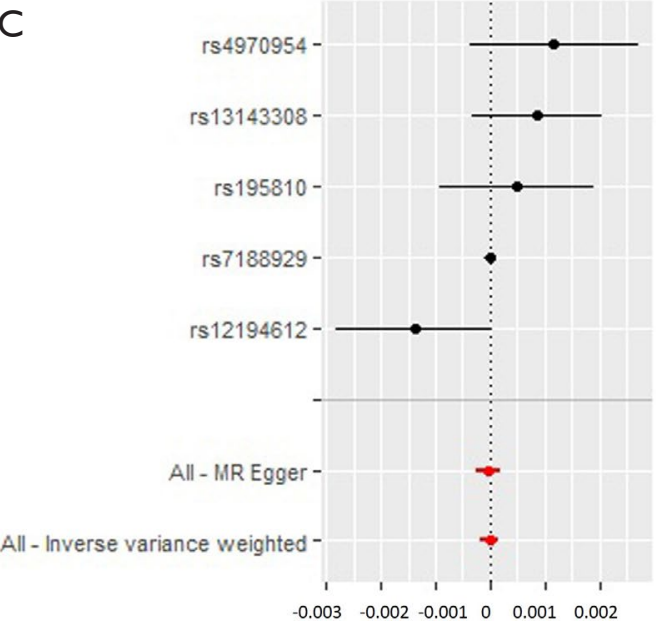

MR effect size for cardiomyopathy (finn-a-19_CARDMYO) on KSD (ukb-b- 13537)

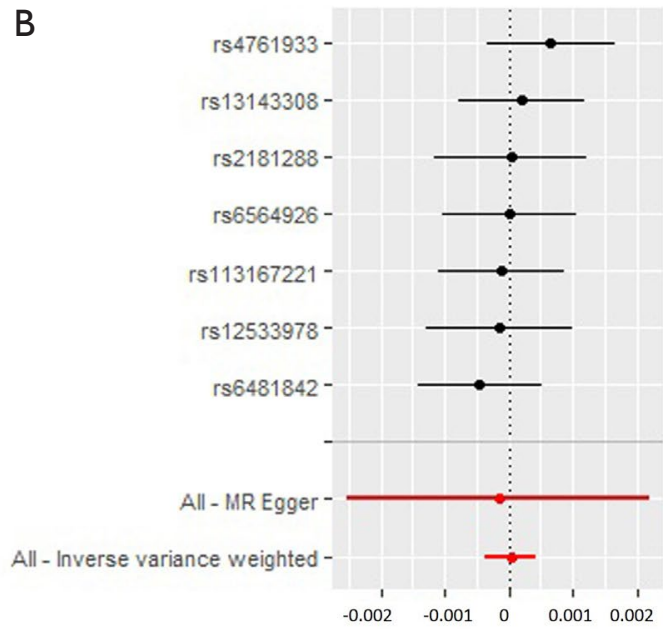

MR effect size for cardiomyopathy (finn-a-I9 CAEDMYOOTH) on KSD (ukkb-b-8297)

D

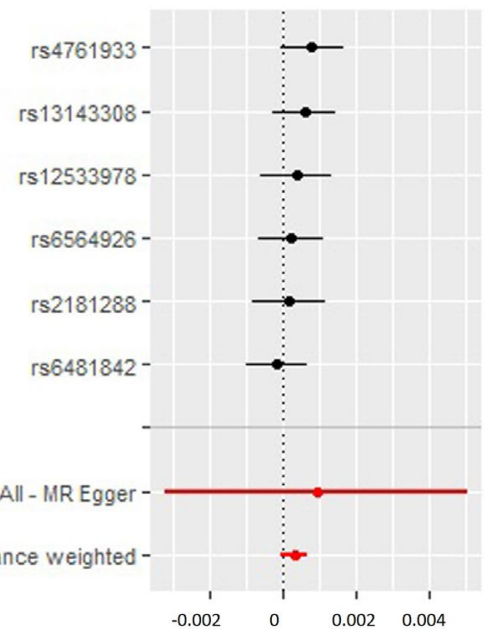

MR effect size for cardiomyopathy (finn-a-I9_CAEDMYOOTH) on KSD (ukb-b-13537)

Figure S1 Mendelian randomization study of the effect of cardiomyopathy on KSD. (A,B) Forest plots were presented for the MR effect size for cardiomyopathy-finn-a-I9_CARDMYO (A) and cardiomyopathy-finn-a-I9_CARDMYOOTH (B) on KSD-ukb-b-8297. (C,D) Forest plots were presented for the MR effect size for cardiomyopathy-finn-a-I9_CARDMYO (C) and cardiomyopathy-finn-a-I9_CARDMYOOTH (D) on KSDukb-b-13537. The results showed that cardiomyopathy didn't affect the outcome of KSD ( $>0.05)$. For the forest plots, red points showing the combined causal estimate using all SNPs together in a single instrument, using each of two different methods [inverse-variance weighted (IVW) and MR-Egger]. Horizontal lines denote $95 \%$ confidence intervals. 